\title{
2-Arylhydrazononitriles in heterocyclic synthesis: a novel route to 1,3-diaryl-1,2,4-triazol-5-amines via a Tiemann rearrangement of arylhydrazonoamidoximes
}

\author{
Hamad M. Al-Matar, ${ }^{\text {a* }}$ Sayed M. Riyadh, ${ }^{a}$ and Mohamed H. Elnagdi ${ }^{b}$ \\ ${ }^{a}$ Department of Chemistry, Faculty of Science, Kuwait University, Safat 13060 Kuwait, P.O. Box \\ 5969 \\ ${ }^{b}$ Department of Chemistry, Faculty of Science, Cairo University, Giza-Egypt, P.O. Box 12613 \\ E-mail:almatarc60@hotmail.com
}

\begin{abstract}
2-Arylhydrazononitriles react with hydroxylamine hydrochloride in refluxing ethanolic sodium acetate to yield amidoximes that cyclized into 1,2,3-triazol-5-amines or 1,2,4-triazol-5-amines depending on the nature of the substituents on hydrazone linkage. NOE difference experiments could successfully be utilized to distinguish 1,2,3-triazoles from isomeric 1,2,4-triazoles.
\end{abstract}

Keywords: Enaminonitriles; X-ray crystal structure determination, acetylamino-1,2,3-triazol-5amines; NOE difference, Boulton-Katritzky rearrangement

\section{Introduction}

The chemistry of 2-arylhydrazono-3-substituted nitriles has been extensively investigated in our laboratories in the past. ${ }^{1-3}$ Our interest in the chemistry of these compounds has recently been revived and we could show that these compounds are excellent precursors to 4-aminopyrazole-5carboxylic acid derivatives, ${ }^{4,5}$ interesting precursors to phosphodiesterase inhibitors such as sildenafil citrate (viagra) ${ }^{4,5}$ as well as 4-acyl-2-substituted-1,2,3-triazolamines, potential precursors to pharmaceutically active triazoloazines; e.g. 1,4-dihydro-5-(2-propoxyphenyl)-7H1,2,3-triazolo[4,5-d]pyrimidine-7-one (Zaprinast). ${ }^{5,6}$ In conjunction to our interest in the chemistry of these compounds we report here the results of our work that enabled developing simple, efficient, novel routes to both 1,2,4-triazol-5-amines and 2,4-disubstituted-1,2,3-triazol5 -amines. Derivatives of both ring systems are important both in the dye industry ${ }^{7}$ and as potential intermediates in pharmaceutical industry. 8,9 


\section{Results and Discussion}

2-Arylhydrazononitriles are readily obtainable via variety of procedures; ${ }^{10-13}$ the simplest is coupling of active methylene nitriles with aromatic diazonium salts. ${ }^{11}$ In this work we could successfully couple active methylene nitriles 1a-c with aromatic diazonium salts producing the 2-arylhydrazolononitriles 2a-d. Benzyl cyanide (3) failed to couple with aromatic diazonium salts under these conditions. As the $\beta$-carbon in enaminonitriles has recently been shown by us to be sufficiently electrophilic to couple readily with aromatic diazonium salts, ${ }^{14}$ benzyl cyanide (3) was condensed with triethyl orthoformate and piperidine following a recently published procedure. ${ }^{5}$ The so formed 2-phenyl-3-piperidinoacrylonitrile (4) coupled smoothly with aromatic diazonium salts yielding 2-arylhydrazononitriles $\mathbf{2 e - h}$. It is believed that $\mathbf{5}$ is a nonisolable intermediate as it undergoes ready Japp-Klingemann cleavage ${ }^{15}$ yielding $\mathbf{2 e - h}$ (Scheme $1)$.

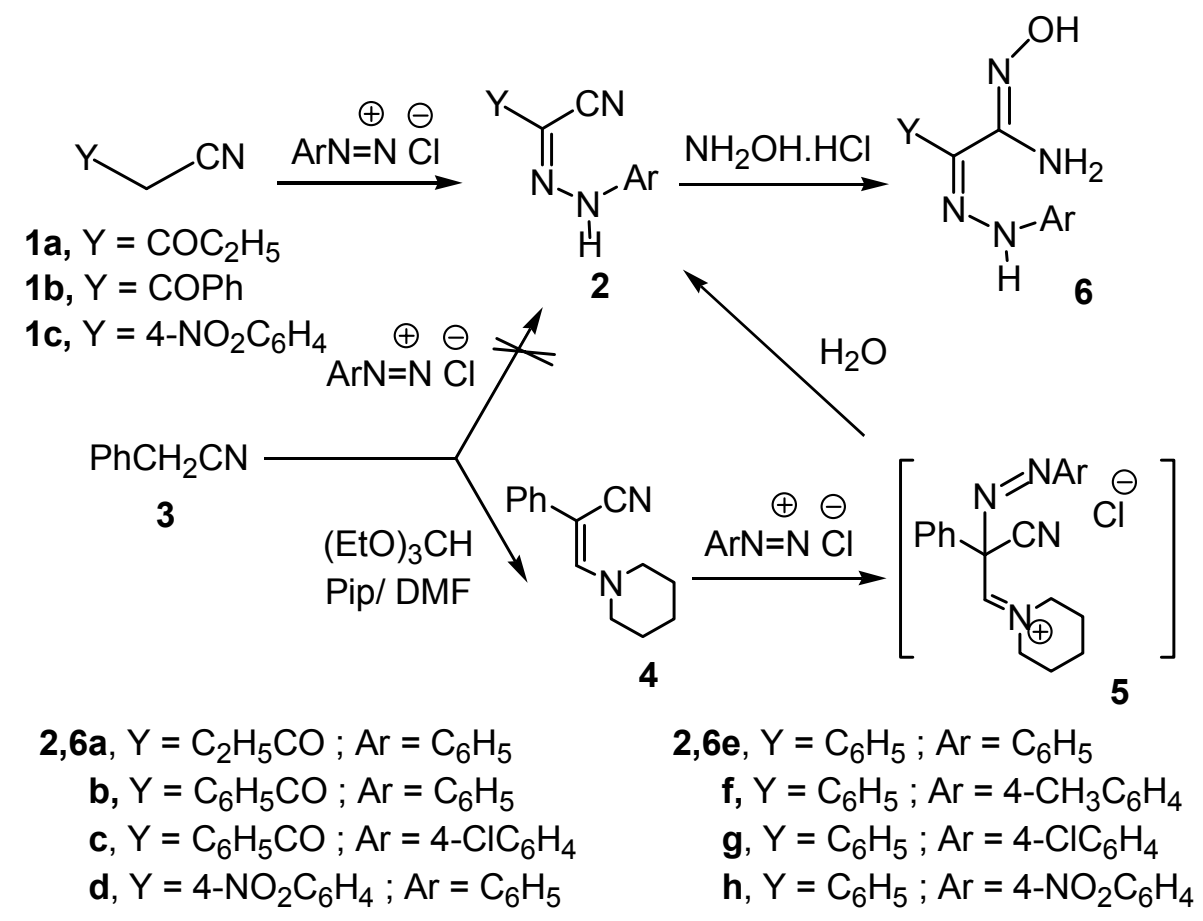

\section{Scheme 1}

Similar to the reported ${ }^{16}$ formation of amidoximes $6 \mathbf{a}-\mathbf{c}$ on reacting $\mathbf{2 a - c}$ with hydroxylamine hydrochloride, the arylhydrazononitriles $\mathbf{2 d - h}$ afforded amidoximes $\mathbf{6 d - h}$ upon treatment with hydroxylamine hydrochloride under similar conditions. Amidoximes 6a-c have been previously cyclized into 1,2,3-triazol-5-amines 7a-c, whose structure was supported by X-ray for 7c, upon reflux in dimethyl formamide in presence of sodium acetate. ${ }^{16}$ However, in our hands, much better yields of 1,2,3-triazol-5-amines were obtained upon replacing sodium acetate by piperidine (Scheme 2). 


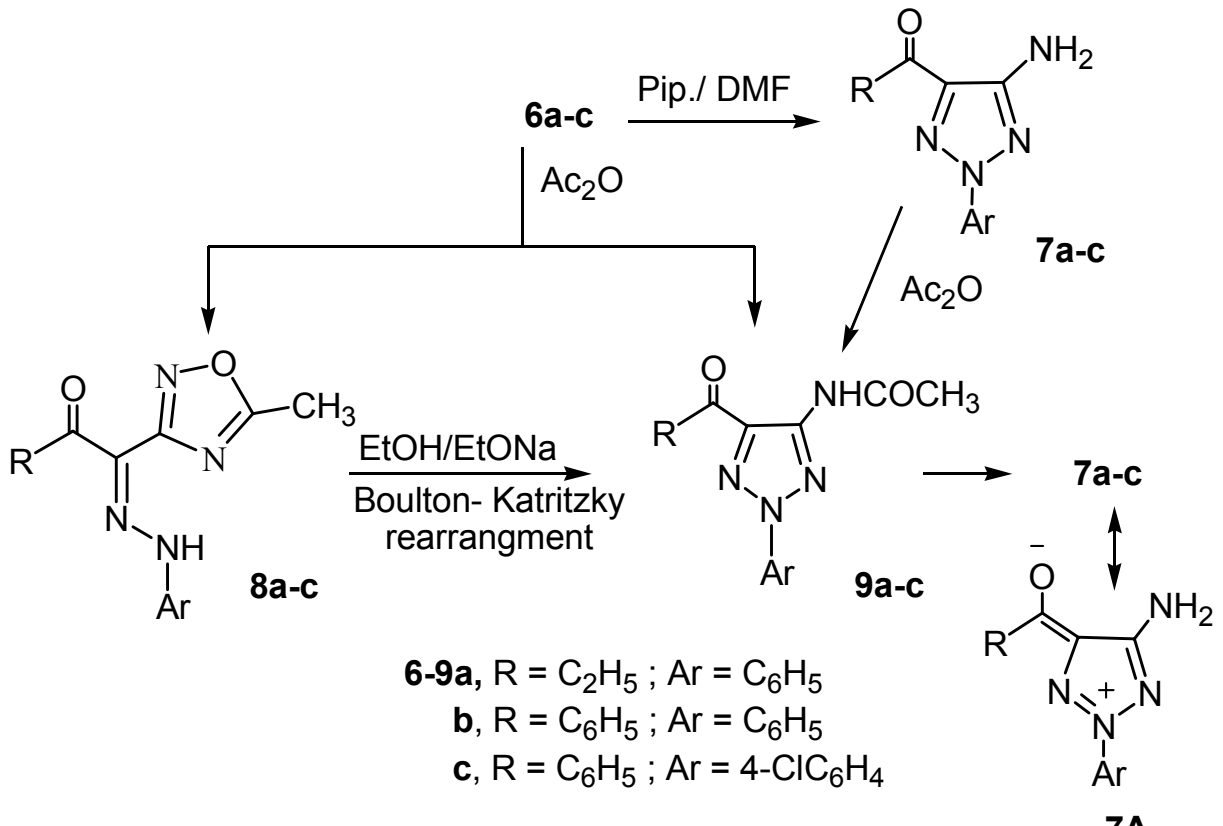

$7 \mathrm{~A}$

\section{Scheme 2}

In an attempt to accelerate cyclization, amidoximes 6a-c were refluxed in acetic anhydride. The obtained products could be assigned the oxadiazolylarylhydrazone structure 8a-c or acetylamino-1,2,3-triazoles 9a-c. Structure 9 could be confirmed for these products based on their identity with acetylamino-1,2,3-triazole 9, prepared via acylating 7a-c with acetic anhydride. Interestingly 9a-c, could be converted into 7a-c upon reflux in ethanolic sodium ethoxide. To verify whether or not an oxadiazole $\mathbf{8}$ is formed initially, amidoximes $\mathbf{6}$ were acylated with acetyl chloride at room temperature, and even under such conditions the only products formed were acetylamino-1,2,3-triazoles 9 (cf. Scheme 2). Thus the intermediacy of oxadiazoles and subsequent Boulton-Katritzky ${ }^{17,18}$ rearrangement, as has recently been assumed, seems least likely. In contrast to the behavior of 6a-c amidoxime $\mathbf{6 d}$ cyclized upon reflux in DMF in presence of piperidine to yield the 1,2,4-triazol-5-amine 11d as was established by Xray crystal structure (cf. Fig. 1). 


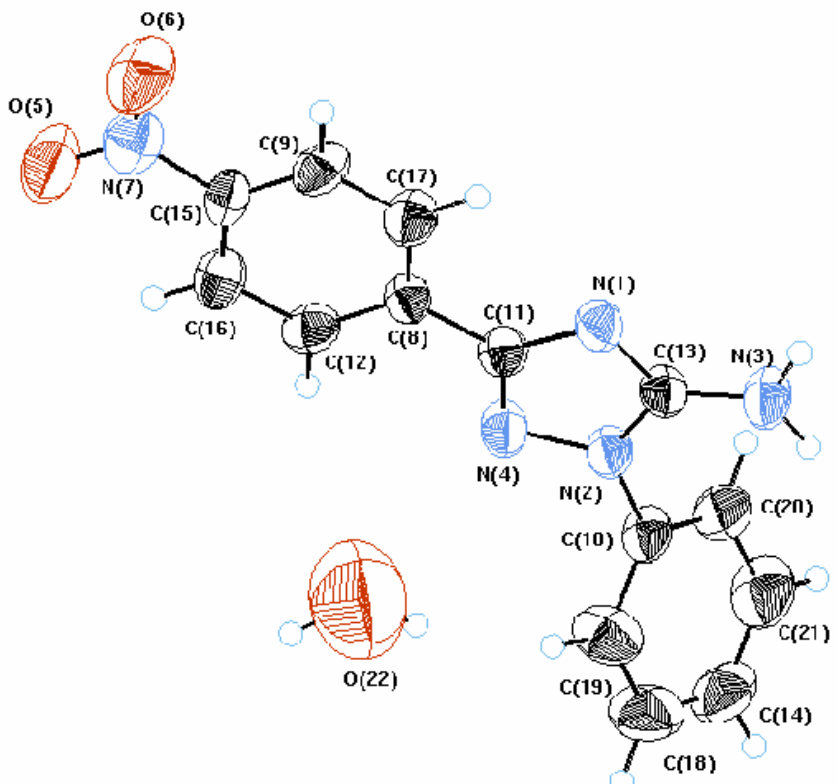

Figure 1. Crystal structure of 3-(4-nitrophenyl)-1-phenyl-1H-1,2,4-triazol-5-amine (11d).

It is believed that amidoxime $\mathbf{6 d}$ in this case has initially undergone a Tiemann rearrangment ${ }^{19-24}$ to yield intermediate 10d that then further cyclized into 11d. It became now clear that we have two competing modes of cyclization that may lead either to 1,2,4-triazole or 1,2,3-triazole amines. It seemed thus mandatory to develop a way to establish, spectroscopically, structure for product of cyclization. An easy way could be achieved through NOE difference experiments. Thus irradiating $\mathrm{NH}_{2}$ protons in $\mathbf{1 1 d}$ at $\delta 6.64 \mathrm{ppm}$ enhanced ortho-aromatic protons at $\delta 7.65 \mathrm{ppm}$ while in the 1,2,3-triazole irradiating amino protons did not effect such enhancement.

Compounds 6e-h also cyclized in refluxing DMF, piperidine yielding the 1,2,4-triazol-5amines 11e-h as indicated from NOE difference experiments (cf. Scheme 3). Conversion of 6 into a 1,2,3-triazole necessitates formation of N-N bond which is an energy demanding process. Aromaticity of the formed 2-substituted-1,2,3-triazole is thus a driving force especially if the substituent can contribute an extra resonance form as in $\mathbf{7 a - c}$ ( $\mathrm{cf}$. form $\mathbf{7 A}$ ).

\section{Conclusions}

Arylhydrazonoamidoximes are readily obtainable compounds that can be easily cyclized to afford in good yields either 1,2,3-triazolamines or 1,2,4-triazolamines depending on the nature of substituent on the hydrazone carbon. A simple spectroscopic method that allows a reliable structure determination of the cyclization product is also suggested. 


\section{Acknowledgements}

The support of this work was received from University of Kuwait through research grant $(\mathrm{SC} 04 / 06)$ and the facilities of Analab/SAF (GS01/01) and (GS03/01) are gratefully acknowledged.

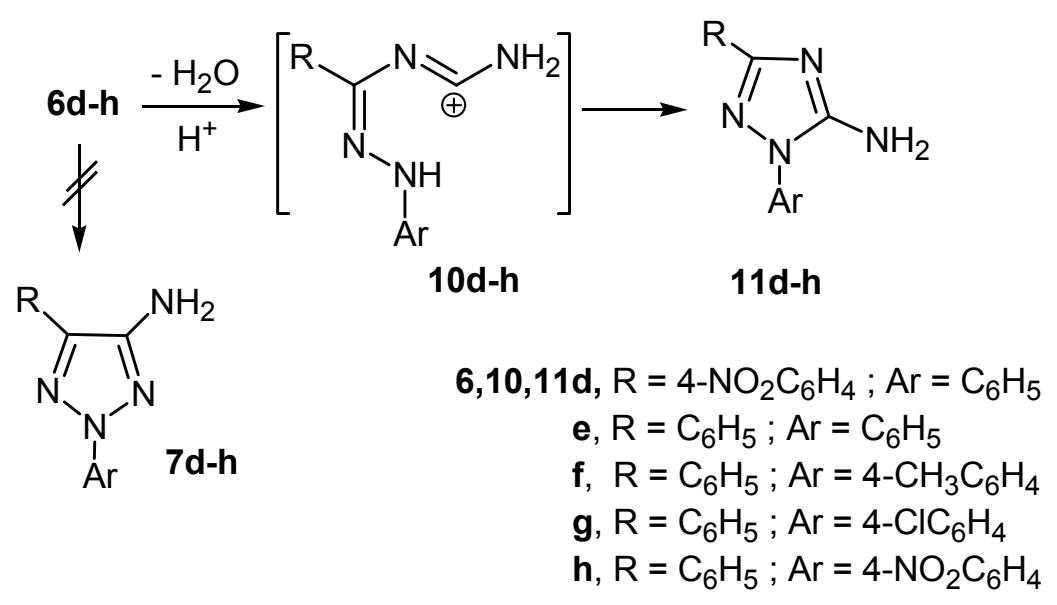

\section{Scheme 3}

\section{Experimental Section}

General Procedures. Melting points were recorded on Gallenkamp apparatus and are uncorrected. Infrared spectra $(\mathrm{KBr})$ were determined on a Perkin-Elmer 2000 FT-IR system. ${ }^{1} \mathrm{H}$ NMR was determined on a Bruker DPX $400 \mathrm{MHz}$ superconducting spectrometer in $\mathrm{CDCl}_{3}$ and DMSO- $\mathrm{d}_{6}$ as solvents and using TMS as internal standard. Mass spectra were measured on MS 30 and MS 9 (AEI) spectrometers, with EI $70 \mathrm{eV}$. Elemental analyses were measured by means of LECO CHNS-932 Elemental Analyzer. Compounds 2a-c, 6a-c and 7a-c were prepared as described in literature. ${ }^{16}$

\section{Crystallographic analysis}

The crystals were mounted on a glass fiber. All measurements were performed on an ENRAF NONIUS FR 590. The data were collected at a temperature of $25{ }^{\circ} \mathrm{C}$ using the $\omega$ scanning technique to a maximum of a $2 \theta$ of $24.108^{\circ}$. The structure was solved by direct method using SIR 92 and refined by full-matrix least squares. Non-hydrogen atoms were refined anisotropically. Hydrogen atoms were located geometrically and were refined isotropically.

\section{Crystal data}

$\mathrm{C}_{14} \mathrm{H}_{11} \mathrm{~N}_{5} \mathrm{O}_{2}, \mathrm{M}=281.275$, monoclinic, $\mathrm{a}=7.9303$ (7), $\mathrm{b}=7.3875$ (7), $\mathrm{c}=23.421$ (3) $\AA, \mathrm{v}=$ $1357.1(2)(\AA)^{3}, \alpha=\gamma=90.00^{\circ}, \beta=98.483(3)^{\circ}$, space group: $\mathrm{P} 2_{1} / \mathrm{c}, \mathrm{D}_{\mathrm{x}}=1.377 \mathrm{Mg} \mathrm{m}^{-3}$ 
reflection 690 measured, $\theta_{\max }=24.09^{\circ}$, $\omega \mathrm{R}$ factor $=0.278$. Figure 1 illustrates the structure as determined. Full data can be obtained on request from the CCDC. ${ }^{25}$

\section{Synthesis of 2-substituted-2-(2-arylhydrazono) acetonitriles (2d-h)}

A cold solution of aryldiazonium salts $(10 \mathrm{mmol})$ was prepared by adding a solution of sodium nitrite (1.4 g dissolved in $10 \mathrm{~mL}$ water) to cold solution of arylamine hydrochloride (10 mmol of arylamine in $6 \mathrm{~mL}, 6 \mathrm{M} \mathrm{HCl}$ ) with stirring. The resulting solution of aryldiazonium salts were then added to a cold solution of either enaminonitrile (4) or acetonitrile derivatives (1) in ethanol $(50 \mathrm{~mL})$ in the presence of sodium acetate trihydrate $(2.8 \mathrm{~g}, 20 \mathrm{mmol})$. The mixture was stirred at room temperature for $1 \mathrm{~h}$ and the solid product was collected by filtration, washed with water and recrystallized from the appropriate solvent.

2-(4-Nitrophenyl)-2-(2-phenylhydrazono)acetonitrile (2d). $1.99 \mathrm{~g}$ (75\%) (green), mp $198{ }^{\circ} \mathrm{C}$ [EtOH]; IR (KBr) v = $3240(\mathrm{NH}), 2208(\mathrm{CN}), 1599(\mathrm{C}=\mathrm{N}) \mathrm{cm}^{-1} ;{ }^{1} \mathrm{H}$ NMR $\left(\mathrm{CDCl}_{3}\right) \delta=7.15-7.46$ (m, 5H, Ar-H), 7.95 (d, 2H, $J=8 H z), 8.33$ (d, 2H, $J=8 \mathrm{~Hz}), 9.10$ (s, 1H, NH); MS, $m / z(\%) 266$ $\left(\mathrm{M}^{+}, 80\right), 239$ (10), 91 (100), 77 (40). Anal. Calcd. for $\mathrm{C}_{14} \mathrm{H}_{10} \mathrm{~N}_{4} \mathrm{O}_{2}$ : C, 63.15; H, 3.79; N, 21.04. Found: C, 62.99; H, 4.10; N, 20.92.

2-Phenyl-2-(2-phenylhydrazono)acetonitrile (2e). 1.55g (70\%) (pale yellow), mp $140{ }^{\circ} \mathrm{C}$ $[\mathrm{MeOH}]$; IR $(\mathrm{KBr}) \mathrm{v}=3235(\mathrm{NH}), 2215(\mathrm{CN}), 1601(\mathrm{C}=\mathrm{N}) \mathrm{cm}^{-1} ;{ }^{1} \mathrm{H}$ NMR $\left(\mathrm{CDCl}_{3}\right) \delta=7.06-$ 7.84 (m, 10H, Ar-H), 8.84 (s, 1H, NH); MS, m/z (\%) 221 (M+100), 194 (80), 91 (90), 77 (60). Anal. Calcd. for $\mathrm{C}_{14} \mathrm{H}_{11} \mathrm{~N}_{3}:$ C, 76.00; H, 5.01; N, 18.99. Found: C, 75.88; H, 5.12; N, 18.77.

2-Phenyl-2-[2-(4-methylphenyl)hydrazono]acetonitrile (2f). 1.69g (72\%) (yellow), mp $120{ }^{\circ} \mathrm{C}$ $[\mathrm{MeOH}] ; \mathrm{IR}(\mathrm{KBr}) \mathrm{v}=3252(\mathrm{NH}), 2207(\mathrm{CN}), 1612(\mathrm{C}=\mathrm{N}) \mathrm{cm}^{-1} ;{ }^{1} \mathrm{H}$ NMR $\left(\mathrm{CDCl}_{3}\right) \delta=2.36(\mathrm{~s}$, $3 \mathrm{H}, \mathrm{Ar}-\mathrm{CH}_{3}$ ), 7.16-7.83 (m, 9H, Ar-H), 8.78 (s, 1H, NH); MS, m/z (\%) $235\left(\mathrm{M}^{+}, 90\right), 105$ (100), 91 (25), 77 (50). Anal. Calcd. for $\mathrm{C}_{15} \mathrm{H}_{13} \mathrm{~N}_{3}$ : C, 76.57; H, 5.57; N, 17.86. Found: C, 76.64; H, $5.72 ; \mathrm{N}, 17.93$.

2-[2-(4-Chlorophenyl)hydrazono]-2-phenyl acetonitrile (2g). 1.99g (78\%) (pale orange), mp $168{ }^{\circ} \mathrm{C}[\mathrm{EtOH}]$; IR $(\mathrm{KBr}) \mathrm{v}=3258(\mathrm{NH}), 2209(\mathrm{CN}), 1601(\mathrm{C}=\mathrm{N}) \mathrm{cm}^{-1} ;{ }^{1} \mathrm{H} \mathrm{NMR}\left(\mathrm{CDCl}_{3}\right) \delta=$ 7.21-7.48 (m, 5H, Ar-H), 7.69 (d, 2H, $J=8 H z), 7.81$ (d, 2H, $J=8 H z), 8.79$ (s, 1H, NH); MS, $m / z(\%) 257\left(\mathrm{M}^{+}+2,40\right), 256\left(\mathrm{M}^{+}+1,50\right), 255\left(\mathrm{M}^{+}, 100\right), 125$ (90), 111 (20), 77 (15). Anal. Calcd. for $\mathrm{C}_{14} \mathrm{H}_{10} \mathrm{ClN}_{3}$ : C, 65.76; H, 3.94; N, 16.43. Found: C, 65.57; H, 3.83; N, 16.43.

2-[2-(4-Nitrophenyl)hydrazono]-2-phenylacetonitrile (2h). 2.13g (80\%) (pale yellow), mp 214 ${ }^{\circ} \mathrm{C}[\mathrm{EtOH}]$; IR $(\mathrm{KBr}) \mathrm{v}=3250(\mathrm{NH}), 2216(\mathrm{CN}), 1598(\mathrm{C}=\mathrm{N}) \mathrm{cm}^{-1} ;{ }^{1} \mathrm{H} \mathrm{NMR}\left(\mathrm{CDCl}_{3}\right) \delta=7.46-$ $7.64(\mathrm{~m}, 5 \mathrm{H}, \mathrm{Ar}-\mathrm{H}), 7.83$ (d, 2H, $J=8 \mathrm{~Hz}), 8.23$ (d, 2H, $J=8 \mathrm{~Hz}), 11.90$ (s, 1H, NH); MS, $m / z$ (\%) $266\left(\mathrm{M}^{+}, 100\right), 239$ (40), 105 (20), 77 (10). Anal. Calcd. for $\mathrm{C}_{14} \mathrm{H}_{10} \mathrm{~N}_{4} \mathrm{O}_{2}$ : C, 63.15; H, 3.79; N, 21.04. Found: C, 63.12; H, 3.80; N, 21.11.

\section{Synthesis of $\boldsymbol{N}$-hydroxy-2,2-disubstituted-acetamidines (6c-h)}

A mixture of 2-arylhydrazonoacetonitrile (2) $(10 \mathrm{mmol})$, hydroxylamine hydrochloride $(0.69 \mathrm{~g}$, $10 \mathrm{mmol})$ and sodium acetate anhydrous $(3 \mathrm{~g})$ in ethanol $(20 \mathrm{~mL})$ was heated under reflux for 3

$\mathrm{h}$. The reaction mixture was poured on water, collected by filtration and recrystallized from the appropriate solvent. 
2-[(4-Chlorophenyl)hydrazono]- $\boldsymbol{N}$-hydroxy-3-oxo-3-phenyl-propionamidine $\quad(6 \mathrm{c}) . \quad 2.37 \mathrm{~g}$ (75\%) (yellow), mp $226{ }^{\circ} \mathrm{C}\left[\mathrm{H}_{2} \mathrm{O} / \mathrm{EtOH}\right]$; IR $(\mathrm{KBr}) \mathrm{v}=3488(\mathrm{OH}), 3436,3276,3175\left(\mathrm{NH}_{2}\right.$, $\mathrm{NH}), 1625(\mathrm{CO}), 1599(\mathrm{C}=\mathrm{N}) \mathrm{cm}^{-1} ;{ }^{1} \mathrm{H} \mathrm{NMR}\left(\mathrm{CDCl}_{3}\right) \delta=6.50\left(\mathrm{~s}, 2 \mathrm{H}, \mathrm{NH}_{2}\right), 7.01-7.54(\mathrm{~m}, 5 \mathrm{H}$, $\operatorname{Ar}-\mathrm{H}), 7.61(\mathrm{~d}, 2 \mathrm{H}, J=9 \mathrm{~Hz}), 7.75$ (d, 2H, $J=9 \mathrm{~Hz}), 8.11(\mathrm{~s}, 1 \mathrm{H}, \mathrm{NH}), 13.49$ (s, 1H, OH); MS, $m / z(\%) 316\left(\mathrm{M}^{+}, 20\right), 299(40), 105$ (100), 77 (80).

$\mathrm{N}$-Hydroxy-2-(4-nitrophenyl)-2-(phenylhydrazono)-acetamidine (6d). 1.79g (61\%) (red), mp $215{ }^{\circ} \mathrm{C}\left[\mathrm{H}_{2} \mathrm{O} / \mathrm{MeOH}\right]$; IR $(\mathrm{KBr}) v=3499(\mathrm{OH}), 3437,3314,3180\left(\mathrm{NH}_{2}, \mathrm{NH}\right), 1650,1598$ $(2 \mathrm{C}=\mathrm{N}) \mathrm{cm}^{-1} ;{ }^{1} \mathrm{H} \mathrm{NMR}\left(\mathrm{CDCl}_{3}\right) \delta=5.70\left(\mathrm{~s}, 2 \mathrm{H}, \mathrm{NH}_{2}\right), 7.18-8.41(\mathrm{~m}, 9 \mathrm{H}, \mathrm{Ar}-\mathrm{H}), 10.98(\mathrm{~s}, 1 \mathrm{H}$, NH), 12.92 (s, 1H, OH); MS, m/z (\%) 299 (M+100), 284 (20), 239 (30), 91 (90), 77 (55). Anal. Calcd. for $\mathrm{C}_{14} \mathrm{H}_{13} \mathrm{~N}_{5} \mathrm{O}_{3}$ : C, 56.18; H, 4.38; N, 23.40. Found: C, 56.02; H, 4.26; N, 23.62.

$\mathrm{N}$-Hydroxy-2-phenylhydrazono-2-phenyl-acetamidine (6e). $1.77 \mathrm{~g}(70 \%)$ (yellow), mp $160{ }^{\circ} \mathrm{C}$ $[\mathrm{MeOH}]$; IR $(\mathrm{KBr}) \mathrm{v}=3497(\mathrm{OH}), 3381,3329,3215\left(\mathrm{NH}_{2}, \mathrm{NH}\right), 1651,1609(2 \mathrm{C}=\mathrm{N}) \mathrm{cm}^{-1} ;{ }^{1} \mathrm{H}$ $\operatorname{NMR}\left(\mathrm{CDCl}_{3}\right) \delta=4.48\left(\mathrm{~s}, 2 \mathrm{H}, \mathrm{NH}_{2}\right), 7.01-7.81(\mathrm{~m}, 10 \mathrm{H}, \mathrm{Ar}-\mathrm{H}), 8.21(\mathrm{~s}, 1 \mathrm{H}, \mathrm{NH}), 11.01(\mathrm{~s}, 1 \mathrm{H}$, $\mathrm{OH})$; MS, $m / z$ (\%) $254\left(\mathrm{M}^{+}, 100\right), 221$ (40), 91 (40), 77 (45). Anal. Calcd. for $\mathrm{C}_{14} \mathrm{H}_{14} \mathrm{~N}_{4} \mathrm{O}: \mathrm{C}$, 66.13 ; H, 5.55; N, 22.03. Found: C, 66.08; H, 5.61; N, 22.12.

$\mathbf{N}$-Hydroxy-2-[(4-methylphenyl)hydrazono]-2-phenyl-acetamidine $\quad(6 f) . \quad 1.88 \mathrm{~g} \quad(70 \%)$ (yellow), mp $152{ }^{\circ} \mathrm{C}[\mathrm{MeOH}]$; IR $(\mathrm{KBr}) v=3498(\mathrm{OH}), 3392,3337,3226\left(\mathrm{NH}_{2}, \mathrm{NH}\right), 1650$, $1613(2 \mathrm{C}=\mathrm{N}) \mathrm{cm}^{-1} ;{ }^{1} \mathrm{H} \mathrm{NMR}\left(\mathrm{CDCl}_{3}\right) \delta=2.34\left(\mathrm{~s}, 3 \mathrm{H}, \mathrm{Ar}-\mathrm{CH}_{3}\right), 4.50\left(\mathrm{~s}, 2 \mathrm{H}, \mathrm{NH}_{2}\right), 6.91-7.58(\mathrm{~m}$, 9H, Ar-H), 7.66 (s, 1H, NH), 10.68 (s, 1H, OH); MS, m/z (\%) 268 (M+1 100), 234 (35), 106 (45), 91 (30), 77 (30). Anal. Calcd. for $\mathrm{C}_{15} \mathrm{H}_{16} \mathrm{~N}_{4} \mathrm{O}$ : C, 67.15; H, 6.01; N, 20.88. Found: C, 67.08; H, $6.08 ; \mathrm{N}, 20.62$.

$\mathbf{N}$-Hydroxy-2-[(4-chlorophenyl)hydrazono]-2-phenyl-acetamidine $\quad \mathbf{( 6 g )} . \quad 1.87 \mathrm{~g} \quad(60 \%)$ (yellow), mp $148{ }^{\circ} \mathrm{C}\left[\mathrm{H}_{2} \mathrm{O} / \mathrm{MeOH}\right]$; IR (KBr) $\mathrm{v}=3499(\mathrm{OH}), 3399,3274,3185\left(\mathrm{NH}_{2}, \mathrm{NH}\right)$, 1641, $1599(2 \mathrm{C}=\mathrm{N}) \mathrm{cm}^{-1} ;{ }^{1} \mathrm{H}$ NMR $\left(\mathrm{CDCl}_{3}\right) \delta=5.75\left(\mathrm{~s}, 2 \mathrm{H}, \mathrm{NH}_{2}\right), 7.16-7.59$ (m, 9H, Ar-H), 7.68 (s, 1H, NH), 12.89 (s, 1H, OH); MS, m/z (\%) $288\left(\mathrm{M}^{+}, 60\right), 253$ (80), 125 (100), 91 (30), 77 (50). Anal. Calcd. for $\mathrm{C}_{14} \mathrm{H}_{13} \mathrm{ClN}_{4} \mathrm{O}$ : C, 58.24; H, 4.54; N, 19.40. Found: C, 58.27; H, 4.63; N, 19.35 .

$\mathbf{N}$-Hydroxy-2-[(4-nitrophenyl)hydrazono]-2-phenyl-acetamidine (6h). $1.79 \mathrm{~g} \quad(60 \%)$ (pale yellow), mp $204{ }^{\circ} \mathrm{C}\left[\mathrm{H}_{2} \mathrm{O} / \mathrm{MeOH}\right]$; IR (KBr) $v=3495(\mathrm{OH}), 3383,3270,3180\left(\mathrm{NH}_{2}, \mathrm{NH}\right)$, 1638, $1601(2 \mathrm{C}=\mathrm{N}) \mathrm{cm}^{-1} ;{ }^{1} \mathrm{H}$ NMR $\left(\mathrm{CDCl}_{3}\right) \delta=6.07\left(\mathrm{~s}, 2 \mathrm{H}, \mathrm{NH}_{2}\right), 7.38-8.16(\mathrm{~m}, 9 \mathrm{H}, \mathrm{Ar}-\mathrm{H})$, 9.92 (s, 1H, NH), 10.76 (s, 1H, OH); MS, m/z (\%) $299\left(\mathrm{M}^{+}, 30\right), 284$ (65), 239 (100), 91 (30), 77 (50). Anal. Calcd. for $\mathrm{C}_{14} \mathrm{H}_{13} \mathrm{~N}_{5} \mathrm{O}_{3}$ : C, 56.18; H, 4.38; N, 23.40. Found: C, 56.12; H, 4.31; N, 23.55 .

\section{Synthesis of $\mathrm{N}$-[2,5-disubstituted-2H-1,2,3-triazol-4-yl]acetamide (9a-c)}

Method A. Compound (6) (1 mmol) was dissolved in $20 \mathrm{~mL}$ acetic acid in the presence of few drops of acetic anhydride. The reaction mixture was refluxed for $4 \mathrm{~h}$ and poured into ice-water mixture, extracted with chloroform $(3 \mathrm{X} 10 \mathrm{ml})$. The organic extracts were dried over anhydrous magnesium sulfate, and evaporated under reduced pressure. The residue was purified through preparative thin layer chromatography [eluent: hexane / AcOEt (3:1)] 
Method B. Compound (6) (1 mmol) was treated with acetyl chloride $(0.079 \mathrm{~g}, 1 \mathrm{mmol})$ in piperidin $(10 \mathrm{~mL})$ and the reaction mixture was stirred for $4 \mathrm{~h}$ at room temperature and triturated as in method $\mathrm{A}$.

$\boldsymbol{N}$-[2-Phenyl-5-propionyl-2H-1,2,3-triazol-4-yl]acetamide (9a). 0.18g (72\%) (yellow), mp 176 ${ }^{\circ} \mathrm{C}$; IR (KBr) $v=3268(\mathrm{NH}), 1712,1675(2 \mathrm{CO}), 1599(\mathrm{C}=\mathrm{N}) \mathrm{cm}^{-1} ;{ }^{1} \mathrm{H}$ NMR $\left(\mathrm{DMSO}-\mathrm{d}_{6}\right) \delta=1.2$ $(\mathrm{t}, 3 \mathrm{H}, J=7.5 \mathrm{~Hz}), 2.35\left(\mathrm{~s}, 3 \mathrm{H}, \mathrm{CH}_{3}\right), 2.41(\mathrm{q}, 2 \mathrm{H}, J=7.5 \mathrm{~Hz}), 7.21-7.62(\mathrm{~m}, 5 \mathrm{H}, \mathrm{Ar}-\mathrm{H}), 10.51$ (s, 1H, NH); MS, m/z (\%) $258\left(\mathrm{M}^{+}, 100\right), 229$ (60), 201 (50), 77 (35). Anal. Calcd. for $\mathrm{C}_{13} \mathrm{H}_{14} \mathrm{~N}_{4} \mathrm{O}_{2}$ : C, 60.45; H, 5.46; N, 21.69. Found: C, 60.22; H, 5.61; N, 21.72.

$\mathrm{N}$-[5-Benzoyl-2-phenyl-2H-1,2,3-triazol-4-yl] acetamide (9b). 0.21g (70\%) (yellow), mp 196 ${ }^{\circ} \mathrm{C}$; IR $(\mathrm{KBr}) \mathrm{v}=3271(\mathrm{NH}), 1675,1663(2 \mathrm{CO}), 1599(\mathrm{C}=\mathrm{N}) \mathrm{cm}^{-1} ;{ }^{1} \mathrm{H}$ NMR (DMSO-d 6$) \delta=$ $2.15\left(\mathrm{~s}, 3 \mathrm{H}, \mathrm{CH}_{3}\right), 7.21-7.82(\mathrm{~m}, 10 \mathrm{H}, \mathrm{Ar}-\mathrm{H}), 10.71$ (s, 1H, NH); MS, $m / z(\%) 306(\mathrm{M}+, 40), 291$ (80), 105 (50), 77 (100). Anal. Calcd. for $\mathrm{C}_{17} \mathrm{H}_{14} \mathrm{~N}_{4} \mathrm{O}_{2}$ : C, 66.66; H, 4.61; N, 18.29. Found: C, 66.52; H, 4.82; N, 18.36.

$\boldsymbol{N}$-[5-Benzoyl-2-(4-chlorophenyl)-2 $\boldsymbol{H}$-1,2,3-triazol-4-yl]acetamide $\quad$ (9c). $\quad 0.255 \mathrm{~g} \quad(75 \%)$ (yellow), mp $226{ }^{\circ} \mathrm{C}$; IR $(\mathrm{KBr}) \mathrm{v}=3280(\mathrm{NH}), 1689,1671(2 \mathrm{CO}), 1596(\mathrm{C}=\mathrm{N}) \mathrm{cm}^{-1} ;{ }^{1} \mathrm{H}$ NMR $\left(\mathrm{DMSO}_{6}\right) \delta=2.05\left(\mathrm{~s}, 3 \mathrm{H}, \mathrm{CH}_{3}\right), 7.56-7.73(\mathrm{~m}, 5 \mathrm{H}, \mathrm{Ar}-\mathrm{H}), 8.00(\mathrm{~d}, 2 \mathrm{H}, J=9 \mathrm{~Hz}), 8.04(\mathrm{~d}, 2 \mathrm{H}$, $J=9 \mathrm{~Hz}), 10.83(\mathrm{~s}, 1 \mathrm{H}, \mathrm{NH}) ;{ }^{13} \mathrm{C} \mathrm{NMR}\left(\mathrm{DMSO}_{6}\right) \delta=23.81\left(\mathrm{CH}_{3}\right), 121.39,129.68,130.73$, 130.92, 133.67, 134.68, 137.28, 138.44, 138.81, 145.83 (Ar-Cs), 169.15, $187.04(2 \mathrm{C}=\mathrm{O})$; MS, m/z (\%) $340\left(\mathrm{M}^{+}, 50\right), 325$ (100), 105 (80), 77 (40). Anal. Calcd. for $\mathrm{C}_{17} \mathrm{H}_{13} \mathrm{ClN}_{4} \mathrm{O}_{2}$ : C, 59.92; $\mathrm{H}$, 3.85; N, 16.44. Found: C, 59.62; H, 3.82; N, 16.46 .

\section{Synthesis of 1,3-disubstituted-1H-[1,2,4]triazol-5-amines (11d-h)}

A solution of $6(1 \mathrm{mmol})$ in DMF $(20 \mathrm{~mL})$ in presence of piperidin $(2 \mathrm{~mL})$ was refluxed for $4 \mathrm{~h}$. acidified with $\mathrm{HCl} /$ ice mixture. The solid product was collected by filtration and recrystallized from appropriate solvent.

3-(4-Nitrophenyl)-1-phenyl-1H-[1,2,4]triazol-5-amine (11d). $0.195 \mathrm{~g}(68 \%)$ (pale brown), $\mathrm{mp}$ $204{ }^{\circ} \mathrm{C}[\mathrm{EtOH}] ; \mathrm{IR}(\mathrm{KBr}) \mathrm{v}=3465,3332\left(\mathrm{NH}_{2}\right), 1597(\mathrm{C}=\mathrm{N}) \mathrm{cm}^{-1} ;{ }^{1} \mathrm{H}$ NMR $\left(\mathrm{DMSO}-\mathrm{d}_{6}\right) \delta=$ $6.74\left(\mathrm{~s}, 2 \mathrm{H}, \mathrm{NH}_{2}\right), 7.42-7.65(\mathrm{~m}, 5 \mathrm{H}, \mathrm{Ar}-\mathrm{H}), 8.17(\mathrm{~d}, 2 \mathrm{H}, \mathrm{J}=8 \mathrm{~Hz}), 8.31(\mathrm{~d}, 2 \mathrm{H}, \mathrm{J}=8 \mathrm{~Hz}) ;{ }^{13} \mathrm{C}$ NMR (DMSO-d $) \delta=125.11,127.51,128.64,129.90,130.57,137.95,138.46,148.42,156.86$, 157.59; MS, $m / z(\%) 281\left(\mathrm{M}^{+}, 100\right), 239$ (50), 91 (90), 77 (25). Anal. Calcd. for $\mathrm{C}_{14} \mathrm{H}_{11} \mathrm{~N}_{5} \mathrm{O}_{2}$ : C, 59.78; H, 3.94; N, 24.90. Found: C, 59.61; H, 3.88; N, 24.85.

1,3-Diphenyl-1H-[1,2,4]triazol-5-amine (11e). $0.165 \mathrm{~g}$ (70\%) (dark yellow), mp $175{ }^{\circ} \mathrm{C}$ $[\mathrm{EtOH}] ;$ IR $(\mathrm{KBr}) \mathrm{v}=3430,3307\left(\mathrm{NH}_{2}\right), 1608(\mathrm{C}=\mathrm{N}) \mathrm{cm}^{-1} ;{ }^{1} \mathrm{H}$ NMR $\left(\mathrm{DMSO}_{6}\right) \delta=5.71(\mathrm{~s}$, $\left.2 \mathrm{H}, \mathrm{NH}_{2}\right), 7.61-8.31(\mathrm{~m}, 10 \mathrm{H}, \mathrm{Ar}-\mathrm{H}) ;{ }^{13} \mathrm{C} \mathrm{NMR}\left(\mathrm{DMSO}_{6}\right) \mathrm{d}_{6} \delta=124.81,125.11,125.64,126.42$, 127.07, 129.45, 130.16, 141.22, 150.46, 152.51; MS, m/z (\%) $236\left(\mathrm{M}^{+}, 100\right), 194$ (40), 77 (55). Anal. Calcd. for $\mathrm{C}_{14} \mathrm{H}_{12} \mathrm{~N}_{4}$ : C, 71.17; H, 5.12; N, 23.71. Found: C, 71.09; H, 5.01; N, 23.60.

1-(4-Methylphenyl)-3-phenyl-1H-[1,2,4]triazol-5-amine (11f). 0.175g (70\%) (yellow), mp 160 ${ }^{\circ} \mathrm{C}[\mathrm{MeOH}] ; \mathrm{IR}(\mathrm{KBr}) \mathrm{v}=3455,3317\left(\mathrm{NH}_{2}\right), 1599(\mathrm{C}=\mathrm{N}) \mathrm{cm}^{-1} ;{ }^{1} \mathrm{H} \mathrm{NMR}\left(\mathrm{CDCl}_{3}\right) \delta=2.3(\mathrm{~s}$, $\left.3 \mathrm{H}, \mathrm{Ar}-\mathrm{CH}_{3}\right), 5.81\left(\mathrm{~s}, 2 \mathrm{H}, \mathrm{NH}_{2}\right), 7.11-7.93(\mathrm{~m}, 9 \mathrm{H}, \mathrm{Ar}-\mathrm{H}) ;{ }^{13} \mathrm{C} \mathrm{NMR}\left(\mathrm{CDCl}_{3}\right) \delta=26.61,123.81$, $124.15,124.54,124.92,125.51,125.92,131.27,133.82,143.16,151.53 ; \mathrm{MS}, \mathrm{m} / z(\%) 250\left(\mathrm{M}^{+}\right.$, 
100), 208 (60), 91 (40), 77 (25). Anal. Calcd. for $\mathrm{C}_{15} \mathrm{H}_{14} \mathrm{~N}_{4}$ : C, 71.98; H, 5.64; N, 22.38. Found: C, 71.90; H, 5.41; N, 22.50.

1-(4-Chlorophenyl)-3-phenyl-1 H-[1,2,4]triazol-5-amine (11g). 0.19g (70\%) (orange), mp 148 ${ }^{\circ} \mathrm{C}$; IR $(\mathrm{KBr}) \mathrm{v}=3446,3327\left(\mathrm{NH}_{2}\right), 1599(\mathrm{C}=\mathrm{N}) \mathrm{cm}^{-1} ;{ }^{1} \mathrm{H} \mathrm{NMR}\left(\mathrm{CDCl}_{3}\right) \delta=5.84\left(\mathrm{~s}, 2 \mathrm{H}, \mathrm{NH}_{2}\right)$, $7.16(\mathrm{~d}, 2 \mathrm{H}, J=8 \mathrm{~Hz}), 7.21-7.53(\mathrm{~m}, 5 \mathrm{H}, \mathrm{Ar}-\mathrm{H}), 7.59(\mathrm{~d}, 2 \mathrm{H}, J=8 \mathrm{~Hz}) ;{ }^{13} \mathrm{C} \mathrm{NMR}\left(\mathrm{CDCl}_{3}\right) \delta=$ $124.18,125.82,127.14,127.95,130.18,135.45,136.28,145.11,154.22,156.12 ; \mathrm{MS}, \mathrm{m} / z(\%)$ $272\left(\mathrm{M}^{+}+2,50\right), 270\left(\mathrm{M}^{+}, 20\right), 228$ (60), 125 (100), 77 (25). Anal. Calcd. for $\mathrm{C}_{14} \mathrm{H}_{11} \mathrm{ClN}_{4}$ : C, 62.11; H, 4.10; N, 20.70. Found: C, 61.99; H, 4.01; N, 20.60.

1-(4-Nitrophenyl)-3-phenyl-1H-[1,2,4]triazol-5-amine (11h). 0.20g (70\%) (dark yellow), mp $182{ }^{\circ} \mathrm{C}$; IR $(\mathrm{KBr}) \mathrm{v}=3447,3387\left(\mathrm{NH}_{2}\right), 1598(\mathrm{C}=\mathrm{N}) \mathrm{cm}^{-1} ;{ }^{1} \mathrm{H}$ NMR $\left(\mathrm{CDCl}_{3}\right) \delta=5.75(\mathrm{~s}, 2 \mathrm{H}$, $\left.\mathrm{NH}_{2}\right), 7.45-8.05(\mathrm{~m}, 5 \mathrm{H}, \mathrm{Ar}-\mathrm{H}), 8.07$ (d, 2H, $\left.J=8 \mathrm{~Hz}\right), 8.47(\mathrm{~d}, 2 \mathrm{H}, J=8 \mathrm{~Hz}) ;{ }^{13} \mathrm{C} \mathrm{NMR}\left(\mathrm{CDCl}_{3}\right)$ $\delta=126.45,126.70,127.64,129.56,129.93,130.54,138.05,144.21,145.16,153.50 ; \mathrm{MS}, \mathrm{m} / z$ (\%) $281\left(\mathrm{M}^{+}, 30\right), 239(40), 77$ (100). Anal. Calcd. for $\mathrm{C}_{14} \mathrm{H}_{11} \mathrm{~N}_{5} \mathrm{O}_{2}$ : C, 59.78; H, 3.94; N, 24.90 . Found: C, 59.90; H, 4.01; N, 24.71.

\section{References}

1. Elnagdi, M. H.; Kassab, N. A. L.; Sobhy, M. E. E.; Hamza, M. R.; Wahby, M. U. J. prakt. Chem. 1972, 314, 815.

2. Elnagdi, M. H.; Abd Allah, S. O. J. prakt. chem. 1973, 315, 1009.

3. Elnagdi, M. H.; Elmoghayar, M. R. H.; Fleita, D. H. J. prakt. chem. 1974, 316, 975.

4. Abdel-Motaleb, R. M.; Makhloof, A. A.; Ibrahim, H. M.; Elnagdi, M. H. J. Heterocyclic Chem. 2006, 43, 931.

5. Abdel-Motaleb, R. M.; Makhloof, A. A.; Ibrahim, H. M.; Elnagdi, M. H. J. Heterocyclic Chem. 2007, 44, in press.

6. Wibberley, A.; Nunn, P. A.; Naylor, A. M.; Ramage, A. G. Br. J. Pharmacol. 2002, 136, 399.

7. Heaton, A. The Chemical Industry $2^{\text {nd }}$ edn., Blackie Academics and Professional: London, 1986; p 144-213.

8. Lin, R.; Connolly, P. J.; Huang, S.; Wetter, S. K.; Lu, Y.; Murray, W. V.; Emanuel, S. L.; Gruninger, R. H.; Fuentes-Pesquera, A. R.; Rugg, C. A.; Middleton, S. A.; Jolliffe, L. K. J. Med. chem. 2005, 48, 4208.

9. Nathanson, J. A. Eur. J. Pharmacol. 1998, 147, 155.

10. Oresmaa, L.; Kotikoski, H.; Haukka, M.; Salminen, J.; Oksala, O.; Pohjala, E.; Moilanen, E.;

Vapaatalo, H.; Vainiotalo, P.; Aulaskari, P. J. Med. Chem. 2005, 48, 4231.

11. Elnagdi, M. H.; Fleita, D. H.; El-Moghayar, M. R. H. Tetrahedron 1975, 31, 63.

12. Al-Saleh, B.; El-Apasery, M. A.; Elnagdi, M. H. J. Chem. Res. 2004, 578.

13. Shawali, A. S.; Abdelkader, M. H.; Eltalbawy, F. M. A. Tetrahedron 2002, 58, 2875.

14. Al-Omran, F.; Abdel Khalik, M. M.; Abou- Elkhair, A.; Elnagdi, M. H. Synthesis 1997, 91. 
15. Phillip, R. R. Org. Reactions 1959, 10, 143.

16. (a) Ghozlan, S. A. S.; Abdelhamid, I. A.; Ibrahim, H. M.; Elnagdi, M. H. Arkivoc 2006, $X V$, 53. (b) Anwar, H. F.; Fleita, D. H.; Kolshorn, H.; Meier, H.; Elnagdi, M. H. Arkivoc 2006, $X V, 1$.

17. Ruccia, M.; Vivona, N.; Spinelli, D. Adv. Heterocycl. Chem. 1993, 56, 49.

18. Vivona, N.; Cusmano, G.; Macaluso, G. J. Chem. Soc. Perkin Trans. 1, 1977, 1616.

19. Tiemann, F. Chem. Ber. 1891, 24, 4162.

20. Smith, P. A. S. Molecular Rearrangements Part 1; De Mayo, P., Ed.; Interscience: New York, 1962; pp 564-565.

21. McCarthy, C. G.; Garner, L. A. Rearrangement involving imidic acid derivatives In The Chemistry of Amides and Imidates Patai, S.; Ed.; John Wiley and Sons: New York, 1975; pp 233.

22. Chandrasekhar, S.; Gopalaiah, K. Tetrahedron Lett. 2003, 44, 755.

23. De Luca, L.; Giacomelli, G.; Porcheddu, A. J. Org. Chem. 2002, 67, 6272.

24. Sharghi, H.; Hosseini, M. Synthesis 2002, 1057.

25. Crystal data for 11d (ref. CCDC 619484) can be obtained on request from the director, Cambridge Crystallographic Data Center, 12 Union Road, Cambrige CB2 1EW, UK 